\title{
[18F]-FDG PET/CT of the Pulmonary Benign Metastasizing Leiomyoma in a Breast Cancer Patient: A Case Report
}

\author{
Chun-Hao Kao ${ }^{1}$, Hong-Wei Gao ${ }^{2}$, Kai-Hsiung Ko ${ }^{3}$, Guo-Shiou Liao ${ }^{4}$ and Chi-Jung Tsai ${ }^{1, *(1)}$ \\ 1 Department of Nuclear Medicine, Tri-Service General Hospital and National Defense Medical Center, \\ Taipei City 11490, Taiwan; x510107@hotmail.com \\ 2 Department of Pathology, Tri-Service General Hospital and National Defense Medical Center, \\ Taipei City 11490, Taiwan; gao11290301@yahoo.com.tw \\ 3 Department of Radiological Diagnosis, Tri-Service General Hospital and National Defense Medical Center, \\ Taipei City 11490, Taiwan; m860818@mail.ndmctsgh.edu.tw \\ 4 Department of Surgery, Division of General Surgery, Tri-Service General Hospital and National Defense \\ Medical Center, Taipei City 11490, Taiwan; guoshiou@mail.ndmctsgh.edu.tw \\ * Correspondence: gironggirl@mail.ndmctsgh.edu.tw; Tel.: +886-2-87927374
}

Citation: Kao, C.-H.; Gao, H.-W.; Ko,

K.-H.; Liao, G.-S.; Tsai, C.-J.

[18F]-FDG PET/CT of the Pulmonary

Benign Metastasizing Leiomyoma in

a Breast Cancer Patient: A Case

Report. Surgeries 2021, 2, 231-236.

https://doi.org/10.3390/

surgeries2030023

Academic Editor: Cornelis F. M. Sier

Received: 5 June 2021

Accepted: 25 June 2021

Published: 28 June 2021

Publisher's Note: MDPI stays neutral with regard to jurisdictional claims in published maps and institutional affiliations.

Copyright: (c) 2021 by the authors. Licensee MDPI, Basel, Switzerland. This article is an open access article distributed under the terms and conditions of the Creative Commons Attribution (CC BY) license (https:// creativecommons.org/licenses/by/ $4.0 /)$.

\begin{abstract}
F-fluorodeoxyglucose ([18F]-FDG) positron emission tomography/computed tomography $(\mathrm{PET} / \mathrm{CT})$ is a useful functional image technique to evaluate the disease extent in many cancers, as well as differentiate benign disease from malignant metastasis. We report the case of a 49-year-old woman with breast cancer and suspected lung metastasis by conventional images. After FDG-PET/CT demonstrating faint, or even no FDG uptake in bilateral pulmonary nodules, accompanied with obvious uterine fibroids, benign metastasizing leiomyoma (BML) of the lung was included in a differential diagnosis and finally confirmed by surgical pathology. FDG PET/CT showed its value in the diagnosis of BML in the breast cancer patient who was misleading as lung metastasis during outpatient follow-up.
\end{abstract}

Keywords: [18F]-FDG PET/CT; breast cancer; pulmonary nodules; benign metastasizing leiomyoma

\section{Introduction}

Benign metastasizing leiomyoma (BML) of lung is a rare disease and may be misdiagnosed with another disease, including malignancy [1]. When the pulmonary nodules are found accidentally in women with breast cancer, metastatic disease should be considered first because the lung is a common metastatic site in breast cancer [2]. However, distinguishing the benign etiology from malignant metastasis by conventional anatomical imaging is challenging [3]. Whole-body $18 \mathrm{~F}$-fluorodeoxyglucose ([18F]-FDG) positron emission tomography/computed tomography (PET/CT) is especially advantageous to help differentiate benign and malignant pulmonary lesions in breast cancer patients and provide whole-body information to rule out other occult disease. Herein, we report a case with a history of breast cancer presented in multiple nodules in the both lungs during regular follow up, which showed slight- and non-FDG-uptake by FDG PET/CT. The pathological results of the pulmonary nodule confirmed the diagnosis of benign metastasizing leiomyoma.

\section{Case}

Our case was a 49-year-old woman with a history of invasive ductal carcinoma of the left breast, pT1aN1a, cM0, estrogen receptor (ER)-positive; progesterone receptor (PR)positive; human epidermal growth factor receptor 2 (Her2)-negative. She underwent left nipple-sparing mastectomy and adjuvant chemotherapy with epirubicin, cyclophosphamide and docetaxel. One year after the treatment, her chest radiography and CT revealed multiple bilateral pulmonary nodules, suspected breast cancer with pulmonary metastasis. An [18F]-FDG PET/CT scan was performed for tumor restaging (Figure 1). The 
maximum intensity projection image and fused PET/CT showed faint and non-FDG avid nodules in the lungs with the maximum size being $1.7 \mathrm{~cm}$ over the right lower lobe, and a maximum standardized uptake value (SUVmax) of 1.3. Additionally, a large mass about $19 \times 16 \mathrm{~cm}^{2}$ in size arising from the uterus with calcified spots occupying the abdominal cavity depicting mild FDG uptake was observed, with an SUVmax 2.2, suggestive of uterine fibroids. Benign metastasizing leiomyomas (BML) in the lung were first impressed.
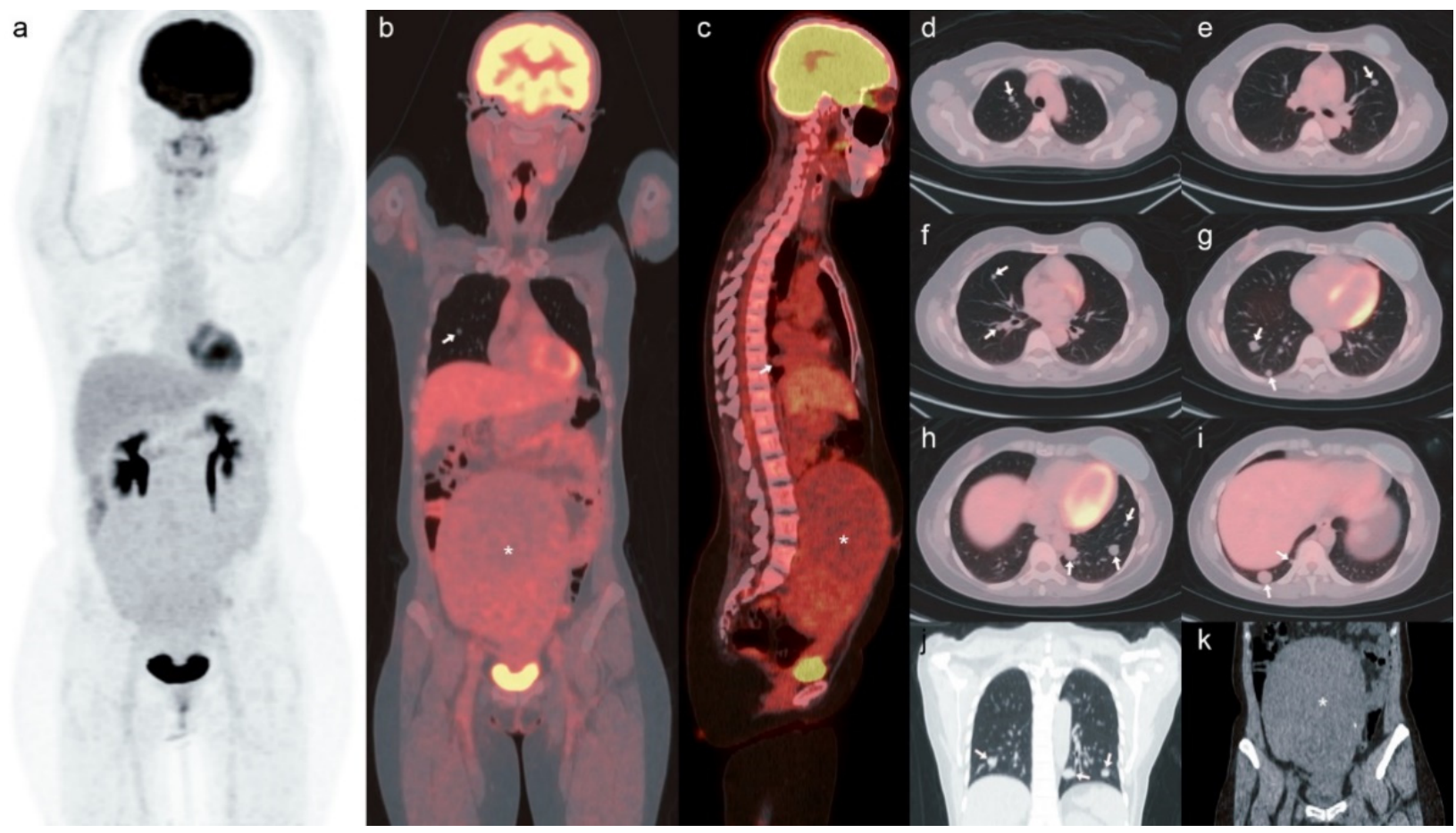

Figure 1. Whole-body [18F]-FDG PET/CT scan. The (a) maximum intensity projection image and the (b) coronal view, (c) sagittal view, (d-i) axial view of fused PET/CT showed faint and non-FDG avid nodules in the lungs (shown by arrows). (j) Coronal view of chest part, attenuation correction CT revealed multiple nodules (shown by arrows) with maximum size of $1.7 \mathrm{~cm}$ over the right lower lobe, and maximum standardized uptake value (SUVmax) of 1.3. (b,c) and (k) Coronal view of abdominal part, attenuation correction CT showed a huge mass about $19 \times 16 \mathrm{~cm}^{2}$ in size arising from uterus with calcified spots occupying the abdominal cavity depicting mild FDG uptake, SUVmax 2.2 (shown by asterisk).

Video-assisted thoracic surgery with a wedge resection of the right lower lobe was performed for pathological confirmation, which revealed one whitish firm solid tumor measuring $1.5 \times 1.5 \times 1.5 \mathrm{~cm}^{3}$ in size (Figure 2). Microscopically (Figure 3), the sections showed pictures of leiomyoma of the pulmonary tissue, characterized by a well-defined nodular lesion composed of smooth muscle proliferation. Immunohistochemistry (IHC) stains showed positive expressions for alpha-smooth actin, caldesmon, ER and PR, negative expressions for proto-oncogene c-kit (CD117) and paired box gene 8 (PAX-8). Positive expression for thyroid transcription factor-1 (TTF-1) was noted only on pneumocytes. The final diagnosis was uterine leiomyoma with pulmonary metastasis. 


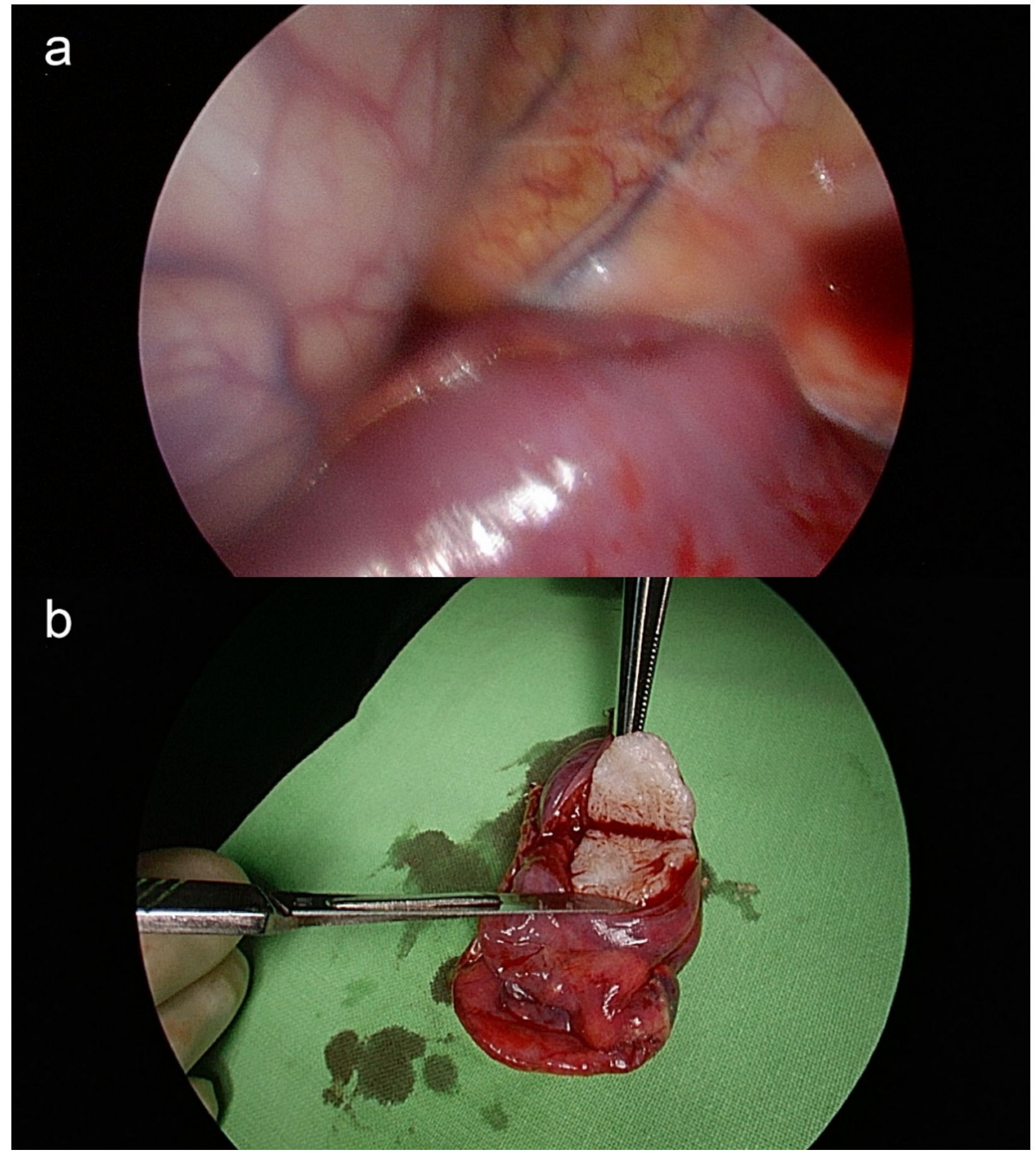

Figure 2. Intraoperative photography. The (a) tumor in the right lower lobe of lung showed (b) whitish firm solid tumor measuring $1.5 \times 1.5 \times 1.5 \mathrm{~cm}^{3}$ in size. 


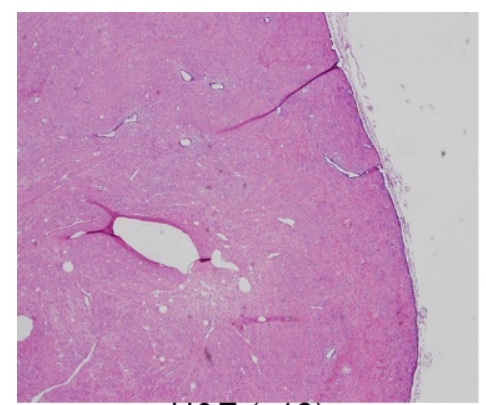

a $H \& E(x 10)$
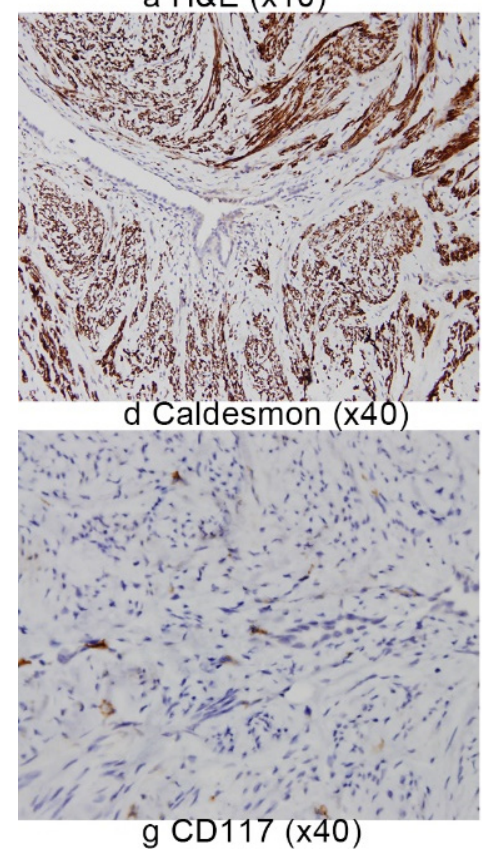

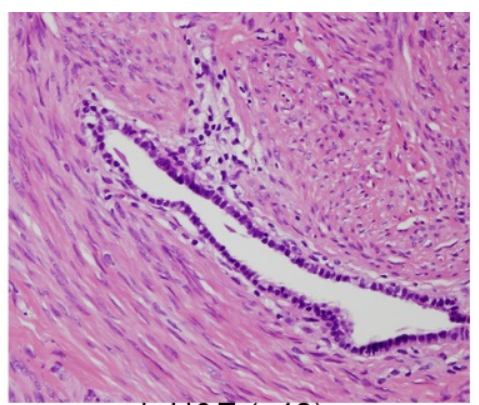

b H\&E $(x 40)$

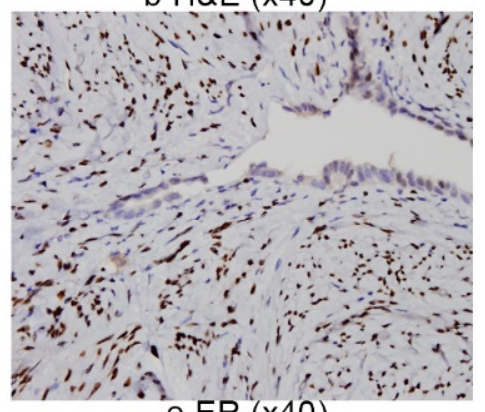

e ER $(x 40)$

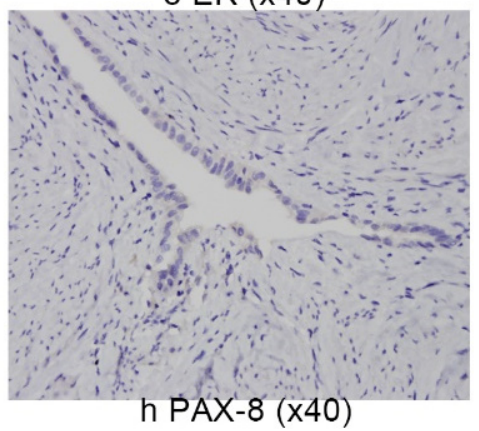

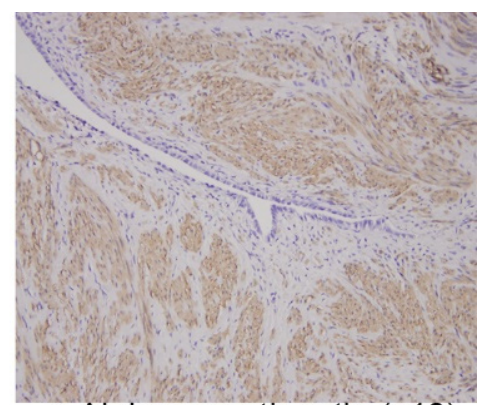

c Alpha-smooth actin (x40)
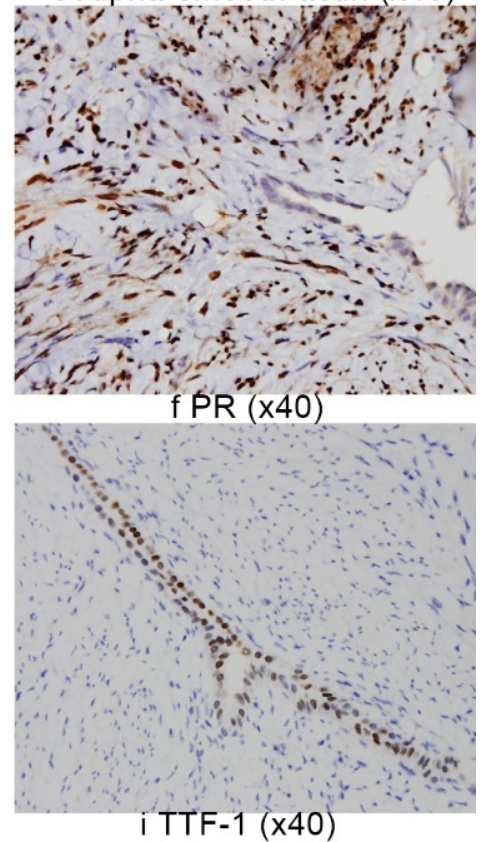

Figure 3. Hematoxylin and eosin (H\&E) and immunohistochemistry (IHC) stains of the specimen from the pulmonary nodule. (a,b) H\&E stains showed a well-defined nodular lesion composed of smooth muscle proliferation. IHC stains showed positive expressions for (c) alpha-smooth actin, (d) caldesmon, (e) estrogen receptor and (f) progesterone receptor, negative expressions for (g) proto-oncogene c-kit (CD117) and (h) paired box gene 8 (PAX-8). Positive expression for (i) thyroid transcription factor-1 (TTF-1) was noted only on pneumocytes.

\section{Discussion}

Pulmonary nodules are seen in various pulmonary diseases, including tumor/metastasis, tuberculosis and infection, but BML is infrequently considered as a candidate in the differential diagnosis of such lesions.

BML is characterized by benign soft-tissue tumors which are strongly associated with uterine leiomyoma and metastasize to other organs [1]. Distant metastasis may occur anywhere, such as in the heart, skeletal muscle, lymph nodes, vascular channels, and bones, but it is most seen to the lungs, and may be mistaken for other malignant metastatic cancer. It is predominantly found in premenopausal women previously diagnosed with uterine leiomyoma, even post operation of hysterectomy or myomectomy [4]. Nucci et al. described consistent chromosomal aberrations (19q and $22 q$ terminal deletions) in five BML cases and significant genetic abnormalities were shared by both lesions from the uterus and lungs [5]. Besides, some studies believed that smooth muscle cells were transferred by blood vessels from the trauma of myomectomy or hysterectomy [6], while there was no history of the abdominal operation in our case. Patients with pulmonary BML are usually asymptomatic and it is discovered incidentally by chest image study. Clinical symptoms may be presented due to the increased size or number of tumors. The standard treatment 
of BML is not established, nor completed. Hormone therapy and surgical intervention may be the therapeutic plans [7-9].

Most BML showed non-FDG or mild-FDG uptake in FDG PET/CT [10,11], and few instances of the disease revealed increased FDG uptake [12]. FDG PET/CT also showed promise in differentiating BML from hypermetabolic malignant disease, such as leiomyosarcoma or other metastatic tumor [13].

Most BML are generally known as slow-growing diseases. Barnaś et al. concluded that the mean time from the previous gynecological surgery to BML diagnosis was 8.8 years in 161 case studies [7]. Kim et al. described the interval of the diagnosis of uterine leiomyoma and BML in 11 case analyses as being between 1 year and 1 month to 19 years [9]. On the other hand, Abu et al. reported a case with a history of uterine fibroid embolization, and the chest CT study showed multiple pulmonary nodules with maximum sizes of $2.2 \mathrm{~cm}$ 2 years later. The nodules were not FDG avid in the following PET/CT study, and BML was diagnosed with histological confirmation [13]. Most BML are asymptomatic, and this might be the reason that increased the duration between the diagnosis of BML and previous chest image study.

There are two case reports of pulmonary BML with breast cancer in Japan $[14,15]$. Ishibashi et al. reported a patient diagnosed with uterine myoma and breast cancer at the age of 32, and multiple pulmonary nodules were noted at the age of 46. Kiyokawa et al. reported the other 60-year-old postmenopausal patient with BML and uterine atypical leiomyoma subsequently, and the surgery for breast cancer was performed 2 years before. To our knowledge, the case we reported is the third case of benign metastasizing leiomyoma of the lung in a breast cancer patient in the English literature, and the first case in which FDG-PET findings are described.

For multiple pulmonary nodules incidentally seen in oncological patients, differential diagnosis should include malignant neoplasm, and pathological confirmation is mandatory. In the current case, our patient was also found to have huge uterine fibroids through wholebody FDG PET/CT, which provided more information to make the optimal diagnosis, such as BML.

\section{Conclusions}

When multiple hypometabolic pulmonary nodules are demonstrated in breast cancer patients accompanying uterine fibroids on PET/CT or with a history of hysterectomy for uterine myoma, BML should be included in the differential diagnosis.

Author Contributions: Conceptualization, C.-H.K. and C.-J.T.; software, C.-H.K.; validation, H.-W.G., K.-H.K. and C.-J.T.; investigation, C.-H.K.; data curation, C.-J.T.; writing-original draft preparation, C.-H.K.; writing-review and editing, G.-S.L. and C.-J.T.; visualization, C.-H.K.; supervision, C.-J.T. All authors have read and agreed to the published version of the manuscript.

Funding: This research received no external funding.

Institutional Review Board Statement: The IRB of our institution waived the need for a patient consent form for this retrospective image study. The study was conducted according to the guidelines of the Declaration of Helsinki.

Informed Consent Statement: The IRB of our institution waived the need for a patient consent form for this retrospective image study.

Data Availability Statement: The data presented in this study are available on request from the corresponding author.

Conflicts of Interest: The authors declare no conflict of interest. 


\section{References}

1. Pacheco-Rodriguez, G.; Taveira-DaSilva, A.M.; Moss, J. Benign Metastasizing Leiomyoma. Clin. Chest Med. 2016, 37, 589-595. [CrossRef] [PubMed]

2. Medeiros, B.; Allan, A.L. Molecular Mechanisms of Breast Cancer Metastasis to the Lung: Clinical and Experimental Perspectives. Int. J. Mol. Sci. 2019, 20, 2272. [CrossRef] [PubMed]

3. Abbott, G.F.; Vlahos, I. CT Diagnosis and Management of Focal Lung Disease. In Diseases of the Chest, Breast, Heart and Vessels 20192022: Diagnostic and Interventional Imaging; Hodler, J., Kubik-Huch, R.A., von Schulthess, G.K., Eds.; Springer: Berlin/Heidelberg, Germany, 2019; pp. 47-55.

4. Asumu, H.; Estrin, Y.; Mohammed, T.L.; Verma, N. Benign Metastasizing Leiomyoma. Curr. Probl. Diagn. Radiol. 2017, 46, 257-259. [CrossRef]

5. Nucci, M.R.; Drapkin, R.; Dal Cin, P.; Fletcher, C.D.; Fletcher, J.A. Distinctive cytogenetic profile in benign metastasizing leiomyoma: Pathogenetic implications. Am. J. Surg. Pathol. 2007, 31, 737-743. [CrossRef] [PubMed]

6. Yuan, X.; Sun, Y.; Jin, Y.; Xu, L.; Dai, H.; Wang, J.; Zhang, Z.; Chen, X. Multiple organ benign metastasizing leiomyoma: A case report and literature review. J. Obstet. Gynaecol. Res. 2019, 45, 2132-2136. [CrossRef]

7. Barnaś, E.; Książek, M.; Raś, R.; Skręt, A.; Skręt-Magierło, J.; Dmoch-Gajzlerska, E. Benign metastasizing leiomyoma: A review of current literature in respect to the time and type of previous gynecological surgery. PLoS ONE 2017, 12, e0175875. [CrossRef] [PubMed]

8. Awonuga, A.O.; Shavell, V.I.; Imudia, A.N.; Rotas, M.; Diamond, M.P.; Puscheck, E.E. Pathogenesis of benign metastasizing leiomyoma: A review. Obstet. Gynecol. Surv. 2010, 65, 189-195. [CrossRef] [PubMed]

9. Kim, H.-S.; Yoon, G.; Lee, J.-S.; Han, J.; Song, S.Y.; Kim, B.-G.; Bae, D.-S.; Lee, J.-W. Pulmonary benign metastasizing leiomyoma: Clinical and therapeutic analyses of 11 patients treated at a single institution. Int. J. Clin. Exp. Med. 2016, 9, $19654-19663$.

10. Sawai, Y.; Shimizu, T.; Yamanaka, Y.; Niki, M.; Nomura, S. Benign metastasizing leiomyoma and 18-FDG-PET/CT: A case report and literature review. Oncol. Lett. 2017, 14, 3641-3646. [CrossRef] [PubMed]

11. Wei, W.T.; Chen, P.C. Benign metastasizing leiomyoma of the lung: A case report and literature review. Oncol. Lett. 2015, 10, 307-312. [CrossRef] [PubMed]

12. Zong, D.; He, W.; Li, J.; Peng, H.; Chen, P.; Ouyang, R. Concurrent benign metastasizing leiomyoma in the lung and lumbar spine with elevated standardized uptake value level in positron-emission tomography computed tomography: A case report and literature review. Medicine 2018, 97, e11334. [CrossRef] [PubMed]

13. Abu Saadeh, F.; Riain, C.O.; Cormack, C.M.; Gleeson, N. Lung metastases from benign uterine leiomyoma: Does 18-FDG-PET/CT have a role to play? Ir. J. Med. Sci. 2019, 188, 619-624. [CrossRef] [PubMed]

14. Kiyokawa, H.; Omiya, H.; Takami, K.; Sekimoto, M.; Mori, K. Metastasizing Leiomyoma of the Lung Detected on Chest X-ray after Surgery for Breast Cancer; Report of a Case. Kyobu Geka 2015, 68, 1103-1106. [PubMed]

15. Ishibashi, H.; Ohta, S.; Hirose, M.; Nakada, C.; Arai, K.; Etou, H. Pulmonary benign metastasizing leiomyoma from uterine myoma; report of a case. Kyobu Geka 2006, 59, 951-954. [PubMed] 